\title{
Front door to post-take: an interdepartmental quality improvement project to enhance patient journeys from emergency department triage through to the medical post-take review
}

\author{
Authors: Darryl E Bernstein, ${ }^{\mathrm{A}}$ Dana Li, ${ }^{\mathrm{A}}$ Abhishek Sharma ${ }^{\mathrm{A}}$ and Ajitha Jayaratnam ${ }^{\mathrm{A}}$
}

\section{Introduction}

Ninety-five per cent of patients attending the emergency department (ED) should be treated or admitted within 4 hours of arrival. ${ }^{1}$ Subsequently, a patient requiring hospital admission must receive consultant review within 14 hours of arrival. ${ }^{2,3}$ In April 2019, only $81.2 \%$ of patients at Watford General Hospital were treated or admitted within 4 hours. A QI project was designed to provide in-depth analysis of the patient journey from ED triage to post-take consultant review, thereby identifying areas for improvement. This report summarises the results of the plan, do, study, act (PDSA) cycle undertaken to improve this pathway over a 4-month period.

\section{Materials and methods}

The first PDSA cycle analysed 40 patients referred to the medical take during 10 weekday shifts in March and April 2019. Data were collected on arrival time, triage time, diagnostic investigations, time until medical assessment, referral time and time until post-take consultant review. Patients were subdivided into those referred to the medical team following ED clinical assessment and those referred following ED triaging without ED clinical assessment. As part of ongoing quality improvement, the ED team implemented an intervention to specifically select diagnostics according to patient need to reduce time to diagnostic results. Further analysis of 20 patients was performed in June 2019.

\section{Results and discussion}

Initial data showed $79.2 \%$ of patients were treated or admitted within 4 hours of ED arrival; $87 \%$ of patients received post-take consultant review within 12 hours. Steps that contributed to delays in decision to admit were blood tests (64 minutes) and imaging requests (103 minutes). Subgroup analysis identified that patients directly referred to the medical team from ED triaging received post-take consultant review faster than those referred following ED clinical assessment (338 vs 448 minutes).

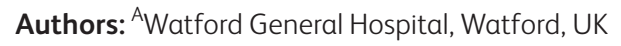

Following ED intervention, $73.7 \%$ of patients were treated or admitted within 4 hours of arrival. $68.4 \%$ of patients received post-take consultant review within 12 hours. Analysis highlighted continued delay in diagnostics: blood tests (63 minutes) and imaging requests (127 minutes). Nevertheless, patients directly referred to the medical team from ED triaging consistently waited less time for post-take consultant review versus those referred following ED clinical assessment (348 vs 633 minutes).

\section{Conclusion}

Using QI methodology, the patient journey from ED triage to review by a senior decision-maker from the medical team was studied to identify areas for improvement in the experience of patients referred to the medical team. Data demonstrated that patients referred from ED triage to the medical team received consultant review faster than those referred following ED clinical assessment. ED intervention to reduce the number of diagnostic tests done at triage, to reduce referral delays, had no impact on improving time to senior speciality review for medical patients. Significant delays remain in diagnostics at the front door and more work with pathology and radiology is needed.

As part of a take pilot being run by the medical team, patients for medical review are being identified earlier at ED triage and directly assessed by specialty medical teams, aiming to improve time from ED triage to senior medical review.

\section{Conflicts of interest}

None declared.

\section{References}

1 Baker C. Accident and emergency statistics: demand, performance and pressure. House of Commons Library, 2017.

2 NHS England. NHS services, seven days a week. NHS, 2013. www. england.nhs.uk/wp-content/uploads/2013/12/forum-summaryreport.pdf

3 NHS England. Seven day services clinical standards. NHS, 2017. www.england.nhs.uk/publication/seven-day-services-clinicalstandards 